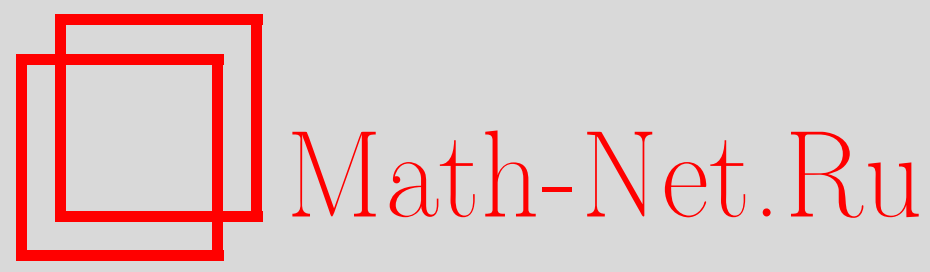

А. Ю. Савин, Б. Ю. Стернин, $\eta$-инвариант и двойственность Понтрягина в $K$-теории, Матем. заметки, 2002, том 71, выпуск 2, 271-291

DOI: https://doi.org/10.4213/mzm346

Использование Общероссийского математического портала Math-Net.Ru подразумевает, что вы прочитали и согласны с пользовательским соглашением http://www.mathnet.ru/rus/agreement

Параметры загрузки:

IP : 54.198 .55 .26

26 апреля 2023 г., 13:14:27






\title{
$\eta$-ИНВАРИАНТ И ДВОЙСТВЕННОСТЬ ПОНТРЯГИНА \\ В $K$-ТЕОРИИ
}

\author{
А. Ю. Савин, Б. Ю. Стернин
}

В работе исследуется топологический смысл спектрального $\eta$-инварианта АтьиПатоди-Зингера. Показано, что удвоенная дробная часть инварианта вычисляется как индекс зацепления в $K$-теории с ориентирующим пучком многообразия. Из двойственности Понтрягина вытекает невырожденность спаривания, определяемого индексом зацепления. Построен пример нетривиальной дробной части для оператора четного порядка.

Библиография: 24 названия.

\section{Введение}

Гилки заметил [1], что $\eta$-инвариант Атьи-Патоди-Зингера [2] является жестким в классе дифференциальных операторов на замкнутых многообразиях при условии

$$
\operatorname{ord} A+\operatorname{dim} M \equiv 1(\bmod 2) \text {. }
$$

Более точно, дробная часть спектрального $\eta$-инварианта эллиптического самосопряженного дифференциального оператора $A$ в этом случае определяется его главным символом и является гомотопическим инвариантом главного символа.

В таких рамках $\eta$-инвариант геометрических операторов первого порядка на четномерных многообразиях был изучен в работе [3]. Оказалось, что в этом случае инвариант принимает только двоично-рациональные значения. Причем нетривиальные дробные части отличные от $1 / 2$ возникают только на неориентируемых многообразиях. Типичным примером является (самосопряженный) оператор Дирака на многообразиях pin $^{c}$ структурой в касательном расслоении. Такие операторы определены, например, на проективных пространствах $\mathbb{R P}^{2 n}$. В этом случае дробная часть спектрального $\eta$-инварианта является важным инвариантом рin $^{c}$ кобордизма [4]. Ситуация с общими дифференциальными операторами оставалась открытой. Более того, в случае операторов четного порядка на нечетномерных многообразиях до недавнего времени не был выяснен даже вопрос о нетривиальности дробной части $\eta$-инварианта. В настоящей работе эти пробелы заполняются.

Работа выполнена при частичной поддержке Российского фонда фундаментальых исследований, гранты № 99-01-01100, № 99-01-01254 и № 00-01-00161, и Институтом математики Потсдамского Университета. 
При вычислении дробного гомотопического инварианта возникает естественньй вопрос: в каких терминах может быть получена формула для такого инварианта? Одним из кандидатов является выражение инварианта при помощи индексов зацепления [5], [6]. В теории гомологий эти индексы определяют билинейное отображение

$$
\text { Tor } H_{i}(M) \times \operatorname{Tor} H_{n-i-1}(M) \rightarrow \mathbb{Q} / \mathbb{Z}
$$

элементов конечного порядка для ориентированного замкнутого $n$-мерного многообразия. Индекс зацепления имеет многочисленные приложения (см. [7]-[11]).

В настоящей работе по аналогии с теорией гомологий исследуется форма зацепления в $K$-теории и в эллиптической теории (последняя возникла также в работе [12]). Двойственность Понтрягина в $K$-теории позволяет доказать невырожденность формы зацепления. Основным результатом работы является равенство удвоенной дробной части $\eta$-инварианта и индекса зацепления главного символа эллиптического оператора с ориентирующим пучком многообразия. Используя это равенство и свойства формы зацепления, доказывается нетривиальность дробной части $\eta$-инварианта для некоторых операторов четного порядка.

Доказательства указанных результатов основаны на редукции рассматриваемого спектрального инварианта к гомотопическому инварианту размерности подпространств, определяемых псевдодифференциальными проекторами, которьй рассматривался в [13], [14]. Общая формула индекса эллиптических операторов в подпространствах из этих работ позволяет выразить дробную часть функционала размерности через индекс операторов в эллиптической теории с коэффициентами в конечной циклической групе $\mathbb{Z}_{n}$, что вместе с соответствующей теоремой об индексе по модулю $n$ дает выражение рассматриваемого дробного инварианта в топологических терминах.

Коротко о содержании работы. В первом разделе для удобства читателей напоминается связь $\eta$-инварианта и функционала размерности подпространств, определяемых псевдодифференциальными проекторами. Здесь кратко излагаются необходимые результаты работ [13]-[15]. Приводится также выражение дробной части этих функционалов через индекс эллиптических операторов в подпространствах. В начале второго раздела кратко излагаются основные результаты работы [16] о теории индекса по конечному модулю. Оставшаяся часть раздела посвящена доказательству двойственности Понтрягина в $K$-теории. Далее, в третьем разделе вводится спаривание зацепления. В четвертом разделе вычисляется действие антиподальной инволюции в $K$-групах вещественных векторных расслоений. Здесь используется описание [17] этих групп в терминах алгебр Клиффорда. Далее сформулирована основная теорема работы, выражающая удвоенную дробную часть функционала размерности через коэффициент зацепления. Обсуждаются примеры. В частности, на основе “скрещенного произведения" [18] эллиптических операторов построено четное подпространство, на котором функционал размерности принимает значение с ненулевой дробной частью. Это дает положительный ответ на вопрос о нахождении оператора четного порядка с ненулевой дробной частью $\eta$-инварианта, которьй был поставлен Гилки.

Мы признательны проф. П. Гилки за плодотворную дискуссию по полученным результатам. 


\section{1. $\eta$-инвариант и индекс в подпространствах}

1. Пусть $E$ - векторное расслоение на гладком многообразии $M$. Линейное подпространство $\widehat{L} \subset C^{\infty}(M, E)$ назьвается псевдодифференииальным, если оно определяется некоторым псевдодифференциальным проектором $P$ порядка нуль следующим обра3OM:

$$
\widehat{L}=\operatorname{Im} P, \quad P: C^{\infty}(M, E) \rightarrow C^{\infty}(M, E) .
$$

Векторное расслоение $L=\operatorname{Im} \sigma(P) \subset \pi^{*} E$ на многообразии косфер $S^{*} M$ назьвается символом подпространства.

Рассмотрим на кокасательном расслоении $T^{*} M$ антиподальную инволюцию

$$
\alpha: T^{*} M \rightarrow T^{*} M, \quad \alpha(x, \xi)=(x,-\xi) .
$$

Подпространство $\widehat{L} \subset C^{\infty}(M, E)$ называется четным (нечетнылм), если его символ $L$ инвариантен (антиинвариантен) относительно инволюции:

$$
L=\alpha^{*} L \quad \text { или } \quad L \oplus \alpha^{*} L=\pi^{*} E .
$$

Обозначим через $\widehat{\operatorname{Even}}(M)$ (соответственно $\widehat{\mathrm{Odd}}(M))$ полугруппы всех четных (соответственно нечетных) псевдодифференциальных подпространств относительно операции прямой суммы.

Псевдодифференциальные подпространства можно задавать также при помощи самосопряженных эллиптических операторов. При этом понятие четности подпространств соответствует четности порядка дифференциальных операторов.

ПРЕДЛОЖЕНИЕ 1. Пусть $A$ - эллиптический самосопряженный оператор неотрицательного порядка. Тогда подпространство $\widehat{L}_{+}(A)$, порохсденное собственными векторами оператора $A$ с неотрицательными собственными значениями, является псевдодифференииальным, а его символ $L_{+}(A)$ равен неотрицательному спектральному подрасслоению главного символа $\sigma(A)$ :

$$
L_{+}(A)=L_{+}(\sigma(A)) \in \operatorname{Vect}\left(S^{*} M\right)
$$

Если оператор $А$ является дифференциальным или удовлетворяет более слабому условию

$$
\alpha^{*} \sigma(A)= \pm \sigma(A)
$$

на главный символ, то подпространство $\widehat{L}_{+}(A)$ является четным (нечетным).

ДокАЗАТЕЛЬСТво этого утверждения можно найти в [19] или [14].

На множестве четных подпространств на нечетномерных многообразиях и нечетных на четномерных определен гомотопически инвариантньй функционал, являющийся аналогом понятия размерности конечномерных пространств. 
ТЕОРема 1 [13], [14]. Существует аддитивный гомотопически инвариантный функиионал

$$
d: \widehat{\operatorname{Even}}\left(M^{\text {odd }}\right) \rightarrow \mathbb{Z}\left[\frac{1}{2}\right] \quad u \quad d: \widehat{\operatorname{Odd}}\left(M^{\mathrm{ev}}\right) \rightarrow \mathbb{Z}\left[\frac{1}{2}\right],
$$

который однозначно опредељяется следующими свойствами:

1) (относительная размерность)

$$
d\left(\widehat{L}+L_{0}\right)-d(\widehat{L})=\operatorname{dim} L_{0}
$$

для пары $\widehat{L}+L_{0}, \widehat{L}$ подпространств, отличаюшихся на конечномерное пространство $L_{0}$;

2) (дополнительность)

$$
d(\widehat{L})+d\left(\widehat{L}^{\perp}\right)=0
$$

где $\widehat{L}^{\perp}$ обозначает ортогональное дополнение $к$ подпространству $\widehat{L}$.

Оказьвается, что спектральньй $\eta$-инвариант Атьи-Патоди-Зингера выражается через функционал размерности подпространств.

Теорема 2 [13], [14]. Для неотрицательного спектрального подпространства $\widehat{L}_{+}(A)$ әллиптического самосопряжсенного дифференииального оператора $A$ положительного порядка имеет место формула

$$
\eta(A)=d\left(\widehat{L}_{+}(A)\right)
$$

при условии, что порядок оператора и размерность многообразия имеют противополохние четности.

Равенство (3) имеет место и для псевдодифференциальных операторов при условии их допустимости в смысле [1].

2. Функционал размерности псевдодифференциальных подпространств входит в формулу индекса эллиптических операторов, действующих в подпространствах.

В самом деле, рассмотрим два псевдодифференциальных подпространства $\widehat{L}_{1,2} \subset$ $C^{\infty}\left(M, E_{1,2}\right)$ и псевдодифференциальньй оператор

$$
D: C^{\infty}\left(M, E_{1}\right) \rightarrow C^{\infty}\left(M, E_{2}\right) .
$$

Если вьполнено включение $D \widehat{L}_{1} \subset \widehat{L}_{2}$, то ограничение

$$
D: \widehat{L}_{1} \rightarrow \widehat{L}_{2}
$$

назьвается оператором, действующим в подпространствах. Ограничение

$$
\sigma(D): L_{1} \rightarrow L_{2}
$$

главного символа $\sigma(D)$ на символы подпространств над косферами $S^{*} M$ назьвается символом оператора в подпространствах. Известно, что замыкание оператора (4) по норме пространств Соболева определяет фредгольмов оператор тогда и только тогда, когда символ (5) является әллиптическим, т.е. устанавливает изоморфизм расслоений.

Для эллиптических операторов в подпространствах справедлива следующая формула индекса [13], [14]. 
Teopema 3. Пycmb

$$
\widehat{L}_{1,2} \in \widehat{\operatorname{Even}}\left(M^{\text {odd }}\right) \quad \text { или } \quad \widehat{\operatorname{Odd}}\left(M^{\mathrm{ev}}\right) .
$$

Тогда индекс әллиптического оператора $D: \widehat{L}_{1} \rightarrow \widehat{L}_{2}$ равен

$$
\operatorname{ind}\left(D, \widehat{L}_{1}, \widehat{L}_{2}\right)=\frac{1}{2} \operatorname{ind} \widetilde{D}+d\left(\widehat{L}_{1}\right)-d\left(\widehat{L}_{2}\right),
$$

где в случае нечетных подпространств

$$
\widetilde{D}: C^{\infty}\left(M, E_{1}\right) \rightarrow C^{\infty}\left(M, E_{2}\right)
$$

- обычный (т.е. действующий в сечениях расслоений, определенных на базе $M$ ) әллиптический оператор с главныцм символом $\sigma(\widetilde{D})$, равным

$$
\sigma(\widetilde{D})=\sigma(D) \oplus \alpha^{*} \sigma(D): L_{1} \oplus \alpha^{*} L_{1} \rightarrow L_{2} \oplus \alpha^{*} L_{2}
$$

для четных подпространств

$$
\widetilde{D}: C^{\infty}\left(M, E_{1}\right) \rightarrow C^{\infty}\left(M, E_{1}\right)
$$

- также обычный әллиптический оператор с главным символом $\sigma(\widetilde{D})$, равным

$$
\sigma(\widetilde{D})=\left[\alpha^{*} \sigma(D)\right]^{-1} \sigma(D) \oplus 1: L_{1} \oplus L_{1}^{\perp} \rightarrow L_{1} \oplus L_{1}^{\perp} .
$$

3. Из формулы индекса (7) непосредственно вытекает следующее утверждение.

СледСтвиЕ 1. Удвоенная дробная часть функционала $d$ зависит от символа подпространства $\widehat{L}$ лишь как от әлемента группь $K\left(S^{*} M\right) / K(M)$.

Применим теперь формулу индекса в подпространствах (7) для вычисления дробной части инварианта $d$. Для этого воспользуемся следуюшим свойством четных (нечетных) подпространств.

ТЕОРема 4. Символ подпространства $\widehat{L}$ при условиях четности (6) определяет әлемент 2-кручения в группе $K\left(S^{*} M\right) / K(M)$. Другими словами, для некоторого числа $N$ и расслоения $F \in \operatorname{Vect}(M)$ на базе имеет место изоморфизм

$$
\sigma: 2^{N} L \rightarrow \pi^{*} F, \quad 2^{N} L=\underbrace{L \oplus \cdots \oplus L}_{2^{N} \text { экземпляров }} .
$$


ДоКАЗАТЕЛЬСТво этой теоремы для четных подпространств см. в [1], для нечетных - в [14].

Применим к эллиптическому оператору (в подпространствах)

$$
\widehat{\sigma}: 2^{N} \widehat{L} \rightarrow C^{\infty}(M, F)
$$

с символом (8) формулу индекса (7). Заметим, что в силу (2) пространство сечений расслоения имеет нулевую "размерность" : $d\left(C^{\infty}(M, F)\right)=0$. Таким образом, формула индекса приводит к равенству

$$
\operatorname{ind}\left(\widehat{\sigma}, 2^{N} \widehat{L}, C^{\infty}(M, F)\right)=\frac{1}{2} \text { ind } \widetilde{\widehat{\sigma}}+2^{N} d(\widehat{L}) .
$$

Перенося операторы $\widehat{\sigma}$ и $\widetilde{\sigma}$ под знак индекса, нетрудно получить следующее выражение для удвоенной дробной части значения функционала размерности (cp. с [16]):

$$
\{2 d(\widehat{L})\}=\frac{1}{2^{N}} \bmod 2^{N} \text {-ind }\left[\left(1 \pm \alpha^{*}\right) \widehat{\sigma}\right]
$$

где $\bmod 2^{N}$-ind $D \in \mathbb{Z}_{2^{N}}$ означает индекс фредгольмова оператора $D$, приведенньй по модулю $2^{N}$, а через $\alpha^{*} \widehat{\sigma}$ обозначен оператор

$$
\widehat{\alpha^{*} \sigma}: 2^{N} \widehat{\alpha^{*} L} \rightarrow C^{\infty}(M, F)
$$

Отметим, что индекс оператора (9), а также индекс в формуле (11) как вычет по модулю $2^{N}$ определяется главным символом оператора. В следующем разделе будет показано, как этот индекс может быть вычислен.

\section{2. Индекс по модулю $n$ и двойственность Понтрягина в $K$-теории}

Напомним некоторые сведения о теории индекса по модулю $n$ из работы [16].

1. Фиксируем некоторое натуральное число $n$. Эллиптическим оператором по модулю $n$ назьвается эллиптический оператор вида

$$
D: n \widehat{L}_{1} \oplus C^{\infty}\left(M, E_{1}\right) \rightarrow n \widehat{L}_{2} \oplus C^{\infty}\left(M, F_{1}\right),
$$

где

$$
\widehat{L}_{1} \subset C^{\infty}(M, E), \quad \widehat{L}_{2} \subset C^{\infty}(M, F)
$$

- подпространства. Прямые суммы

$$
n\left(\widehat{L}_{1} \oplus C^{\infty}\left(M, E_{1}\right)\right) \stackrel{n D}{\rightarrow} n\left(\widehat{L}_{2} \oplus C^{\infty}\left(M, F_{1}\right)\right)
$$

будем назьвать тривиальными операторами по модулю $n$. Группа стабильных гомотопических классов эллиптических операторов по модулю тривиальных обозначается через $\operatorname{Ell}\left(M, \mathbb{Z}_{n}\right)$. В работе [16] показано, что эта группа определяет $K$-теорию с коэффициентами в $\mathbb{Z}_{n}$. 
ТЕорема 5. Имеет место изоморфизм групп

$$
\operatorname{Ell}\left(M, \mathbb{Z}_{n}\right) \stackrel{\chi_{n}}{=} K_{c}\left(T^{*} M, \mathbb{Z}_{n}\right)
$$

Здесь $K_{c}-K$-теория с компактными носителями.

Дадим явную формулу для изоморфизма $\chi_{n}$. Прежде всего напомним, что $K$-теория с коэффициентами $\mathbb{Z}_{n}$ определяется при помощи так называемого пространства $\mathrm{My}$ pa $M_{n}$. Это некоторое топологическое пространство с $K$-групшами, равными

$$
\widetilde{K}^{0}\left(M_{n}\right)=\mathbb{Z}_{n}, \quad K^{1}\left(M_{n}\right)=0 .
$$

В качестве пространства Мура можно, например, взять двумерньй комплекс, получаемый из диска $D^{2}$ отождествлением точек на границе по действию группы $\mathbb{Z}_{n}$ :

$$
M_{n}=\left\{D^{2} \subset \mathbb{C}|| z \mid \leqslant 1\right\} /\left\{e^{i \varphi} \sim e^{i(\varphi+2 \pi k / n)}\right\} .
$$

Обозначим векторное расслоение, являющееся образующей группы $\widetilde{K}^{0}\left(M_{n}\right)=\mathbb{Z}_{n}$, через $\gamma_{n}$. Зафиксируем также некоторую тривиализацию $\beta$ прямой суммы $n \gamma_{n}$

$$
n \gamma_{n} \stackrel{\beta}{\rightarrow} \mathbb{C}^{n} .
$$

Для топологического пространства $X$ его $K$-групшы с коэффициентами $\mathbb{Z}_{n}$ определяются по формуле

$$
K^{*}\left(X, \mathbb{Z}_{n}\right)=K^{*}\left(X \times M_{n}, X \times p t\right) .
$$

Далее, произвольньй оператор вида (12) стабильно гомотопен (см. [16]) некоторому оператору

$$
n \widehat{L} \stackrel{D}{\rightarrow} C^{\infty}(M, F),
$$

для которого определено следующее семейство эллиптических символов на многообразии $M$, параметризованное пространством Мура $M_{n}$ :

$$
\begin{aligned}
& \chi_{n}[D] \\
& =\left[\pi^{*} F^{\sigma^{-1}(D)} \rightarrow n L \stackrel{\beta^{-1} \otimes 1_{L}}{\rightarrow} \gamma_{n} \otimes n L \stackrel{1_{\gamma} \otimes \sigma(D)}{\rightarrow} \gamma_{n} \otimes \pi^{*} F\right] \in K_{c}\left(T^{*} M \times M_{n}, T^{*} M \times p t\right)
\end{aligned}
$$

(здесь используется разностная конструкция для эллиптических семейств). Отметим, что индекс эллиптического оператора по модулю $n$ определяется его главным символом как вычет

$$
\bmod n \text {-ind }\left(n \widehat{L} \stackrel{D}{\rightarrow} C^{\infty}(M, F)\right) \in \mathbb{Z}_{n} .
$$

Для этого индекса-вычета справедлива теорема об индексе, которую можно переформулировать в виде коммутативности треугольника

$$
\begin{aligned}
& \operatorname{Ell}\left(M, \mathbb{Z}_{n}\right) \\
& \swarrow \chi_{n} \quad \searrow \bmod n \text {-ind } \quad \\
& K_{c}\left(T^{*} M, \mathbb{Z}_{n}\right) \stackrel{p_{!}}{\longrightarrow} \quad \mathbb{Z}_{n}
\end{aligned}
$$


где через $p_{!}: K_{c}\left(T^{*} M, \mathbb{Z}_{n}\right) \rightarrow \mathbb{Z}_{n}$ обозначено отображение прямого образа в $K$-теории с коэффициентами $\mathbb{Z}_{n}$.

Ниже будет использована точная последовательность, связьвающая эллиптические операторы по модулю $n$ с обычными эллиптическими операторами.

Для этого обозначим группу стабильных гомотопических классов эллиптических операторов через $\operatorname{Ell}(M)$ (см. [18]), а группу стабильных гомотопических классов псевдодифференциальных подпространств через $\operatorname{Ell}_{1}(M)$ (см. [16]).

Отображения, сопоставляющие операторам и подпространствам их главные символы, осуществляют изоморфизмы с соответствующими $K$-группами

$$
\operatorname{Ell}(M) \stackrel{\chi_{\varrho}}{\rightarrow} K_{c}\left(T^{*} M\right), \quad \operatorname{Ell}_{1}(M) \stackrel{\chi_{1}}{\rightarrow} K_{c}^{1}\left(T^{*} M\right) ;
$$

напомним (см. [2] или [16]), что второе отображение определяется как композиция

$$
\chi_{1}: \operatorname{Ell}_{1}(M) \rightarrow K\left(S^{*} M\right) / K(M) \stackrel{\delta}{\rightarrow} K_{c}^{1}\left(T^{*} M\right),
$$

где сначала подпространству сопоставляется его символ, а затем применяется изоморфизм $\delta$, индуцированньй кограничным отображением в $K$-теории,

$$
\delta: K\left(S^{*} M\right) \rightarrow K_{c}^{1}\left(T^{*} M\right) .
$$

Имеет место коммутативность диаграммы

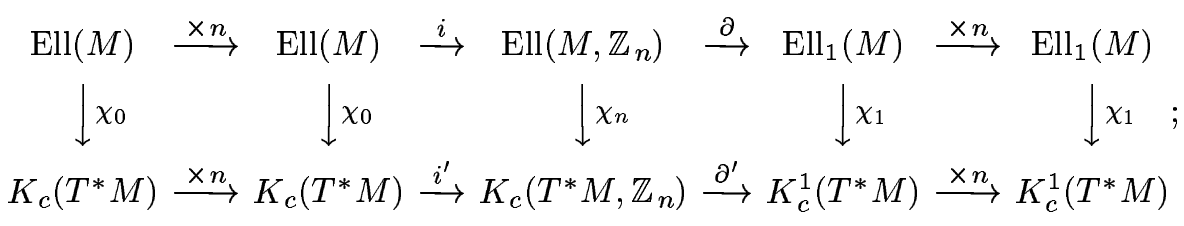

здесь отображения $\times n$ сопоставляют элементу $x$ абелевой группы его кратное $n x$, отображение $i$ индуцировано вложением обычных эллиптических операторов в множество операторов по модулю $n$, а гомоморфизм Бокштейна $\partial$ определяется формулой

$$
\partial\left[n \widehat{L}_{1} \oplus C^{\infty}\left(M, E_{1}\right) \stackrel{D}{\rightarrow} n \widehat{L}_{2} \oplus C^{\infty}\left(M, F_{1}\right)\right]=\left[\widehat{L}_{1}\right]-\left[\widehat{L}_{2}\right] .
$$

Для пары натуральных чисел $n, m$ рассмотрим естественные вложения $\mathbb{Z}_{n} \subset \mathbb{Z}_{m n}$. Прямой предел

$$
\lim _{n \rightarrow \infty} \mathbb{Z}_{n}=\mathbb{Q} / \mathbb{Z}
$$

определяет $K$ - и Ell-группы с коэффициентами в групше $\mathbb{Q} / \mathbb{Z}$ :

$$
K_{c}\left(T^{*} M, \mathbb{Q} / \mathbb{Z}\right)=\lim _{\longrightarrow} K_{c}\left(T^{*} M, \mathbb{Z}_{n}\right), \quad \operatorname{Ell}(M, \mathbb{Q} / \mathbb{Z})=\lim _{\longrightarrow} \operatorname{Ell}\left(M, \mathbb{Z}_{n}\right) .
$$

При этом диаграмма (18) переходит в диаграмму с точньпи строками

$$
\begin{aligned}
& \operatorname{Ell}(M) \quad \rightarrow \quad \operatorname{Ell}(M) \otimes \mathbb{Q} \stackrel{i}{\rightarrow} \operatorname{Ell}(M, \mathbb{Q} / \mathbb{Z}) \stackrel{\partial}{\rightarrow} \operatorname{Ell}_{1}(M) \rightarrow \operatorname{Ell}_{1}(M) \otimes \mathbb{Q} \\
& \downarrow \chi_{0} \quad \downarrow \chi_{0} \otimes 1 \quad \downarrow \chi_{\mathbb{Q} / \mathbb{Z}} \quad \downarrow \chi_{1} \quad \downarrow \chi_{1} \otimes 1 . \\
& K_{c}\left(T^{*} M\right) \rightarrow K_{c}\left(T^{*} M\right) \otimes \mathbb{Q} \stackrel{i^{\prime}}{\rightarrow} K_{c}\left(T^{*} M, \mathbb{Q} / \mathbb{Z}\right) \stackrel{\partial^{\prime}}{\rightarrow} K_{c}^{1}\left(T^{*} M\right) \rightarrow K_{c}^{1}\left(T^{*} M\right) \otimes \mathbb{Q}
\end{aligned}
$$


2. Рассмотрим форму пересечения

$$
K_{c}^{i}\left(T^{*} M, \mathbb{Q} / \mathbb{Z}\right) \times K_{c}^{i}(M) \rightarrow K_{c}^{0}\left(T^{*} M, \mathbb{Q} / \mathbb{Z}\right) \stackrel{p_{1}}{\rightarrow} \mathbb{Q} / \mathbb{Z}
$$


ресечение элементов $x$ и $y$ будем обозначать через $x \cap y$.

Напомним, что спаривание

$$
\langle\cdot, \cdot\rangle: G_{1} \times G_{2} \rightarrow G_{3}
$$

абелевых групп $G_{1}, G_{2}$ со значениями в абелевой группе $G_{3}$ назьвается невырожденным, если отображения

$$
\langle x, \cdot\rangle: G_{2} \rightarrow G_{3} \quad \text { и } \quad\langle\cdot, y\rangle: G_{1} \rightarrow G_{3},
$$

получаемые фиксированием одного из аргументов, являются нулевыми только в случае, когда $x=0$ (соответственно $y=0)$.

ТЕорема 6 (двойственность Понтрягина). Спаривание (20) является невырохсденным. Более того, фиксируя первый аргумент, получаем изоморфизм

$$
K_{c}^{i}\left(T^{*} M, \mathbb{Q} / \mathbb{Z}\right) \simeq \operatorname{Hom}\left(K^{i}(M), \mathbb{Q} / \mathbb{Z}\right) .
$$

ДокАЗАТЕЛЬСТво. Отметим, что невырожденность спаривания следует из изоморфизма (21).

Так же, как и двойственность Пуанкаре (см., например, [20]), двойственность Понтрягина может быть доказана при помощи принципа Майера-Виеториса. Для произвольного открытого множества $U \subset M$ рассмотрим отображение

$$
K_{c}^{i}\left(T^{*} U, \mathbb{Q} / \mathbb{Z}\right) \rightarrow \operatorname{Hom}\left(K^{i}(U), \mathbb{Q} / \mathbb{Z}\right)
$$

Согласно принципу Майера-Виеториса для проверки изоморфности отображения при $U=M$ достаточно

а) проверить его для стягиваемого множества $U$;

б) доказать индуктивный переход: если отображение является изоморфизмом для двух множеств $U, V$ и пересечения $U \cap V$, то оно является изоморфизмом для объединения.

Рассмотрим стягиваемое множество $U$. Тогда

$$
K_{c}^{*}\left(T^{*} U, \mathbb{Q} / \mathbb{Z}\right)=K^{*}(p t)=\mathbb{Q} / \mathbb{Z} \oplus 0
$$

и

$$
K^{*}(U)=\mathbb{Z} \oplus 0
$$

а соответствующее отображение

$$
\mathbb{Q} / \mathbb{Z} \rightarrow \operatorname{Hom}(\mathbb{Z}, \mathbb{Q} / \mathbb{Z})
$$


является изоморфизмом. Справедливость требования а) установлена.

Для доказательства свойства б) рассмотрим диаграмму



в которой верхняя строка является точной последовательностью Майера-Виеториса, а нижняя получается из аналогичной последовательности применением функтора Hom( · , $\mathbb{Q} / \mathbb{Z}$ ) (этот функтор сохраняет точность последовательностей). Вертикальные отображения индуцированы спариванием пересечения. Эта диаграмма является коммутативной с точностью до знака. Предположим, что отображение (22) является изоморфизмом для $U, V$ и пересечения $U \cap V$. В силу леммы о пяти гомоморфизмах, примененной к указанной диаграмме, получаем изоморфность над объединением $U \cup V$. Свойство б) доказано.

Таким образом, оба условия применения принципа Майера-Виеториса вьполнены. Теорема доказана.

ЗАмЕчАнИЕ 1 . Используя $K$-теорию с коэффициентами в топологической групе $\mathbb{R} / \mathbb{Z}$ (ее определение см. в [2]), этим методом можно установить двойственность по Понтрягину групп $K_{c}^{i}\left(T^{*} M, \mathbb{R} / \mathbb{Z}\right)$ и $K^{i}(M)$ :

$$
K_{c}^{i}\left(T^{*} M, \mathbb{R} / \mathbb{Z}\right) \simeq \operatorname{Hom}\left(K^{i}(M), \mathbb{R} / \mathbb{Z}\right), \quad K^{i}(M) \simeq \operatorname{Hom}\left(K_{c}^{i}\left(T^{*} M, \mathbb{R} / \mathbb{Z}\right), \mathbb{R} / \mathbb{Z}\right)
$$

т.е. в этом случае каждая из них является группой характеров другой.

ЗАмечаниЕ 2. Аналогичным методом можно доказать двойственность Понтрягина для произвольного $K$-ориентированного замкнутого многообразия или многообразия с краем (т.е. многообразия $\operatorname{cospin}^{c}$ структурой в касательном расслоении). С этих более общих позиций в теореме 6 получена двойственность Понтрягина-Лефшеца в $K$-теории на почти комплексном многообразии $T^{*} M$ групп с компактными носителями и абсолютных групп.

ЗАмЕчАниЕ 3. Можно также установить невырожденность спаривания

$$
K_{c}^{i}\left(T^{*} M\right) \times K^{i}(M, \mathbb{Q} / \mathbb{Z}) \rightarrow K_{c}^{0}\left(T^{*} M, \mathbb{Q} / \mathbb{Z}\right) \stackrel{p}{\rightarrow} \mathbb{Q} / \mathbb{Z} .
$$

Как и в теории (ко)гомологий (см. [5]), двойственность Понтрягина влечет двойственность Пуанкаре для подгрупп кручения. Билинейная форма, определяющая эту двойственность, называется формой зачепления.

\section{3. Форма зацепления в $K$-теории}

1. Рассмотрим гомоморфизм Бокштейна

$$
\partial: K_{c}^{i}\left(T^{*} M, \mathbb{Q} / \mathbb{Z}\right) \rightarrow K_{c}^{i-1}\left(T^{*} M\right)
$$

(см. диаграмму (19)). Образ этого отображения состоит из элементов конечного порядка. Будем обозначать через Tor $G$ подгруппу элементов конечного порядка абелевой группы $G$. 
ОПРЕДЕЛЕниЕ 1. Формой защепления назьвается спаривание

$$
\begin{aligned}
\cap: \operatorname{Tor} K_{c}^{i-1}\left(T^{*} M\right) \times \operatorname{Tor} K^{i}(M) & \rightarrow \mathbb{Q} / \mathbb{Z}, \\
(x, y) & \mapsto x^{\prime} \cap y,
\end{aligned}
$$

где $x^{\prime} \in K_{c}^{i}\left(T^{*} M, \mathbb{Q} / \mathbb{Z}\right)$ - произвольньй элемент $K$-теории с коэффищиентами такой, что $\partial x^{\prime}=x$, a $x^{\prime} \cap y \in \mathbb{Q} / \mathbb{Z}$ - индекс пересечения из предыдущего раздела.

Так же, как и форма пересечения, форма зацепления определяется умножением

$$
\begin{aligned}
\operatorname{Tor} K_{c}^{i-1}\left(T^{*} M\right) \times \operatorname{Tor} K^{i}(M) & \rightarrow K_{c}\left(T^{*} M, \mathbb{Q} / \mathbb{Z}\right), \\
(x, y) & \mapsto x^{\prime} y, \quad \partial x^{\prime}=x .
\end{aligned}
$$

Лемма 1. Умножсение (23) и форма зацепления корректно определены.

ДокАЗАТЕЛЬСтво. Надо проверить, что случайньй выбор элемента $x^{\prime}$ не приводит к изменению произведения $x^{\prime} y$. Пусть $x=\partial x^{\prime \prime}$. Для разности $x^{\prime}-x^{\prime \prime}$ имеем $\partial\left(x^{\prime}-x^{\prime \prime}\right)=0$. Следовательно, $x^{\prime}-x^{\prime \prime}=i(z)$ для некоторого элемента $z \in K_{c}^{i}\left(T^{*} M\right) \otimes \mathbb{Q}$. Но произведение $z y \in K_{c}^{0}\left(T^{*} M\right) \otimes \mathbb{Q}$ является элементом кручения, поэтому $z y=0$. Таким образом, $x^{\prime} y=x^{\prime \prime} y$. Лемма доказана.

Форму зацепления можно определить аналогичным образом, используя принадлежность второго аргумента подгруппе кручения. Для этого надо рассмотреть гомоморфизм Бокштейна д в коэффищиентной последовательности

$$
\cdots \rightarrow K^{i-1}(M, \mathbb{Q} / \mathbb{Z}) \stackrel{\partial}{\rightarrow} K^{i}(M) \rightarrow K^{i}(M) \otimes \mathbb{Q} \rightarrow \cdots
$$

В качестве индекса зацепления элементов $x \in \operatorname{Tor} K_{c}^{i-1}\left(T^{*} M\right), y \in \operatorname{Tor} K^{i}(M)$ положим

$$
x \cap^{\prime} y=x \cap y^{\prime}
$$

Оказывается, что эти два способа определяют спаривания зацепления, которые отличаются только знаком. А именно, имеет место равенство

$$
x \cap^{\prime} y=(-1)^{\operatorname{deg} x+1} x \cap y .
$$

Указанная формула вытекает из следующего предложения.

ПРЕДЛОЖЕНИЕ 2. Для әлементов $x^{\prime} \in K_{c}^{i}\left(T^{*} M, \mathbb{Q} / \mathbb{Z}\right), y^{\prime} \in K^{j}(M, \mathbb{Q} / \mathbb{Z})$ выполнено равенство

$$
\partial x^{\prime} y^{\prime}=(-1)^{\operatorname{deg} x^{\prime}+1} x^{\prime} \partial y^{\prime}
$$


ДокАЗАТЕЛЬСтво. Будем считать, что элементы $x^{\prime}, y^{\prime}$ происходят из $K$-групп с коэффициентами в группе $\mathbb{Z}_{n}$ для достаточно большого $n$ :

$$
x^{\prime}=I_{*} x_{0}, \quad y^{\prime}=n_{*} y_{0}, \quad x_{0} \in K_{c}^{i}\left(T^{*} M, \mathbb{Z}_{n}\right), \quad y_{0} \in K^{j}\left(M, \mathbb{Z}_{n}\right),
$$

где через $I$ обозначено вложение $\mathbb{Z}_{n} \subset \mathbb{Q} / \mathbb{Z}$.

Рассмотрим точную последовательность

$$
0 \longrightarrow \mathbb{Z}_{n} \stackrel{\times n}{\longrightarrow} \mathbb{Z}_{n^{2}} \stackrel{\bmod n}{\longrightarrow} \mathbb{Z}_{n} \longrightarrow 0
$$

и отвечающую ей последовательность в $K$-теории

$$
\cdots \longrightarrow K_{c}^{i+j}\left(T^{*} M, \mathbb{Z}_{n}\right) \stackrel{\partial^{\prime \prime}}{\longrightarrow} K_{c}^{i+j+1}\left(T^{*} M, \mathbb{Z}_{n}\right) \stackrel{\times n}{\longrightarrow} K_{c}^{i+j+1}\left(T^{*} M, \mathbb{Z}_{n^{2}}\right) \longrightarrow \cdots .
$$

Применим к произведению $x_{0} y_{0} \in K_{c}^{i+j}\left(T^{*} M, \mathbb{Z}_{n}\right)$ гомоморфизм Бокштейна. Имеем

$$
\partial^{\prime \prime}\left(x_{0} y_{0}\right)=\partial x_{0} y_{0}+(-1)^{\operatorname{deg} x_{0}} x_{0} \partial y_{0}
$$

С другой стороны, в силу точности $\times n \circ \partial^{\prime \prime}\left(x_{0} y_{0}\right)=0$. Следовательно, и

$$
I_{*} \partial^{\prime \prime}\left(x_{0} y_{0}\right)=0 \text {. }
$$

Из (24)-(26) получаем требуемое:

$$
\partial x^{\prime} y^{\prime}+(-1)^{\operatorname{deg} x^{\prime}} x^{\prime} \partial y^{\prime}=0 .
$$

ТЕоРема 7 (двойственность Пуанкаре для груп кручения). Форма зацепления

$$
\operatorname{Tor} K_{c}^{i-1}\left(T^{*} M\right) \times \operatorname{Tor} K^{i}(M) \rightarrow \mathbb{Q} / \mathbb{Z}
$$

является невырожденной. В частности, она определяет изоморфизмы

$$
\begin{aligned}
\operatorname{Tor} K_{c}^{i-1}\left(T^{*} M\right) & \simeq \operatorname{Hom}\left(\operatorname{Tor} K^{i}(M), \mathbb{Q} / \mathbb{Z}\right), \\
\operatorname{Tor} K^{i}(M) & \simeq \operatorname{Hom}\left(\operatorname{Tor} K_{c}^{i-1}\left(T^{*} M\right), \mathbb{Q} / \mathbb{Z}\right) .
\end{aligned}
$$

СлЕдСТВИЕ 2. Группы Tor $K_{c}^{i-1}\left(T^{*} M\right)$ u Tor $K^{i}(M)$ являются изоморфными.

Это следует из (неканонического) изоморфизма $G \simeq \operatorname{Hom}(G, \mathbb{Q} / \mathbb{Z})$ для конечной абелевой группы $G$.

ДоКАЗАТЕЛЬСТВо ТЕОРЕМЫ 7. Сначала докажем невырожденность по второму аргументу. Предположим, что $x \cap y=0$ для произвольного $x \in \operatorname{Tor} K_{c}^{i-1}\left(T^{*} M\right)$. Это означает, что для любого $x^{\prime} \in K_{c}^{i}\left(T^{*} M, \mathbb{Q} / \mathbb{Z}\right)$ также получаем $x^{\prime} \cap y=0$. Из двойственности Понтрягина следует, что в этом случае $y=0$.

Невырожденность формы по первому аргументу следует из двойственности Понтрягина, отвечающей форме пересечения

$$
K_{c}^{i-1}\left(T^{*} M\right) \times K^{i}(M, \mathbb{Q} / \mathbb{Z}) \rightarrow \mathbb{Q} / \mathbb{Z}
$$

(см. замечание 3).

Второе утверждение теоремы следует из конечности подгрупп кручения. Теорема доказана. 
2. Изоморфизм эллиптической теории и $K$-теории из первого пункта предыдущего раздела позволяет построить форму зацепления в терминах эллиптических операторов.

ОПРЕДЕЛЕНИЕ 2. Формой зацепления в әллиптической теории назьвается билинейное спаривание

$$
\begin{aligned}
\operatorname{Tor} \operatorname{Ell}_{1}(M) \times \operatorname{Tor} K^{0}(M) & \rightarrow \mathbb{Q} / \mathbb{Z}, \\
(x, y) & \mapsto \text { ind } x^{\prime} y, \quad \partial x^{\prime}=x .
\end{aligned}
$$

ПРЕДЛОЖЕНИЕ 3. Формы зачепления в әллиптической и $K$-теории изоморфны, т.е. следующая диаграмма коммутативна:

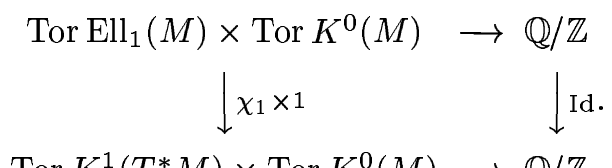

ДоКАЗАТЕЛЬСТВо предложения следует из коммутативности диаграммы



Коммутативность левого квадрата вытекает из изоморфности коэффициентных последовательностей в $K$-теории и в эллиптической теории (см. (19)), а коммутативность правого квадрата составляет формулу для индекса по модулю $n$ (см. (17)). Предложение доказано.

Выпишем явное выражение для формы зацепления в эллиптической теории. Рассмотрим $x=[\widehat{L}], y=\left[G_{1}\right]-\left[G_{2}\right]$ для некоторого псевдодифференциального подпространства $\widehat{L}$ и векторных расслоений $G_{1,2} \in \operatorname{Vect}(M)$. Тогда согласно первому определению для некоторого элемента

$$
x^{\prime}=\left[n \widehat{L} \stackrel{\widehat{\sigma}}{\rightarrow} C^{\infty}(M, F)\right] \in \operatorname{Ell}\left(M, \mathbb{Z}_{n}\right)
$$

индекс зацепления равен

$$
x \cap y=\operatorname{ind} x^{\prime} y=\frac{1}{n} \bmod n \text {-ind }\left(\widehat{\sigma} \otimes 1_{G_{1}-G_{2}}\right),
$$

где через $\widehat{\sigma} \otimes 1_{G_{1}}-G_{2}$ обозначен оператор $\widehat{\sigma}$ с коэффициентами в векторном расслоении $G_{1}-G_{2}$. В соответствии со вторым определением для элемента

$$
y^{\prime}=\left[m G_{1} \stackrel{g}{\rightarrow} m G_{2}\right] \in K^{1}\left(M, \mathbb{Z}_{m}\right)
$$

(см. [2]) этот индекс определяется формулой

$$
x \cap y=\operatorname{ind} x y^{\prime}=\frac{1}{m} \bmod m \text {-ind }\left(1_{\widehat{L}} \otimes g\right),
$$




ЗАмЕчАниЕ 4. Применяя изоморфизм Пуанкаре в комплексной $K$-теории к многообразию $T^{*} M$ (см. [21]), имеем

$$
K_{c}^{1}\left(T^{*} M\right) \simeq K_{1}(M)
$$

следовательно, спаривание зацепления можно рассматривать как невыр ожденное спаривание $K$-гомологий с $K$-когомологиями многообразия $M$ :

$$
\operatorname{Tor} K_{1}(M) \times \operatorname{Tor} K^{0}(M) \stackrel{\cap}{\rightarrow} \mathbb{Q} / \mathbb{Z}
$$

Отметим внешнее сходство формулы (27) для индекса зацепления и формулы (11) для дробной части функционала размерности. Отличие состоит в том, что в последней формуле рассматривается действие антиподальной инволюции, а не умножение на расслоение. В следующем разделе показьвается, что антиподальная инволюция на $K$-группах действует как тензорное умножение на ориентирующий пучок расслоения (cp. с [22] в ориентируемом случае).

\section{4. Антиподальная инволюция и ориентируемость}

Пусть $V$ - вешественное векторное расслоение над компактом $X$. Рассмотрим антиподальную инволюцию

$$
\begin{aligned}
\alpha: V & \rightarrow V, \\
v & \mapsto-v .
\end{aligned}
$$

ТЕорема 8. Индуцированная инволюиия $\alpha^{*}$ в K-теории действует по формуле

$$
\alpha^{*}=(-1)^{n} \Lambda^{n}(V): K_{c}^{*}(V) \rightarrow K_{c}^{*}(V),
$$

где через $\Lambda^{n}(V), n=\operatorname{dim} V$, обозначен ориентирующий пучок расслоения $V$.

ДоКАЗАТЕЛЬСТВО. Без ограничения общности можно считать расслоение $V$ четномерным: $n=\operatorname{dim} V=2 k$. Будем рассматривать также действие инволюции $\alpha^{*}$ только на четных $K$-группах $K_{c}^{0}(V)$ (нечетньй случай сводится к рассматриваемому умножением на одномерное тривиальное расслоение, так как при этом обе части равенства (28) изменяются на -1$)$.

Каруби в [17], [22] было найдено описание элементов групп вида $K_{c}^{0}(V)$ в терминах алгебр Клиффорда. Напомним основные определения. Над пространством $X$ рассмотрим расслоение $\mathrm{Cl}(V)$ алгебр Клиффорда векторного расслоения $V$. Над каждой точкой $x$ базы $X$ алгебра Клиффорда $\mathrm{Cl}\left(V_{x}\right)$ мультипликативно порождена векторным пространством $V_{x}$, при этом выполнены соотношения

$$
v_{1} v_{2}+v_{2} v_{1}=2\left\langle v_{1}, v_{2}\right\rangle
$$

для некоторого фиксированного скалярного произведения $\langle\cdot, \cdot\rangle$ в расслоении $V$.

Будем рассматривать четверки $\left(E, c, f_{1}, f_{2}\right)$, где $E$ - комплексное расслоение над $X$, $c$ - гомоморфизм расслоений

$$
c: \mathrm{Cl}(V) \rightarrow \operatorname{Hom}(E, E),
$$


который является гомоморфизмом расслоений алгебр. Говорят, что $c$ устанавливает на $E$ структуру клиффордова модуля. Инволюции $f_{1}$ и $f_{2}$ расслоения $E$ антикоммутируют с умножением Клиффорда:

$$
f_{i} c(v)+c(v) f_{i}=0, \quad i=1,2 .
$$

На множестве четверок $\left(E, c, f_{1}, f_{2}\right)$ вводится отношение стабильной гомотопической эквивалентности. Соответствующую групу классов обозначим через $K^{V}(X)$. В [17] доказано, что получаемая группа изоморфна групп $K_{c}^{0}(V)$. Для изоморфизма

$$
t: K^{V}(X) \rightarrow K_{c}^{0}(V)
$$

может быть дана следуюшая явная формула

$$
t\left[E, c, f_{1}, f_{2}\right]=\left[\pi^{*} \operatorname{ker}\left(f_{1}-1\right) \stackrel{\left(1-c(v) f_{2}\right)\left(1+c(v) f_{1}\right)}{\longrightarrow} \pi^{*} \operatorname{ker}\left(f_{2}-1\right)\right], \quad \text { где } \pi: S V \rightarrow X .
$$

Здесь через $S V$ обозначено расслоение единичных сфер векторного расслоения $V$, а элемент в правой части равенства понимается в смысле разностной конструкции (см., например, [23]) для относительной группы $K(B V, S V) \simeq K_{c}(V)$.

Из формулы (29) видно, что антиподальная инволюция $\alpha^{*}$ на четверках определяется формулой

$$
\alpha^{*}\left[E, c, f_{1}, f_{2}\right]=\left[E,-c, f_{1}, f_{2}\right] .
$$

Покажем, что четверка $\left[E,-c, f_{1}, f_{2}\right]$ отличается от четверки $\left[E, c, f_{1}, f_{2}\right] \otimes \Lambda^{2 k}(V)$ на изоморфизм векторных расслоений (ср. [3]).

Для этого в каждой тривиализуюшей окрестности расслоения $V$ рассмотрим ортонормированньй базис $e_{1}, e_{2}, \ldots, e_{2 k}$ и определим элемент

$$
\beta=i^{k} c\left(e_{1}\right) \cdots c\left(e_{2 k}\right) .
$$

Нетрудно видеть, что $\beta^{2}=1$ и элемент $\beta$ антикоммутирует с клиффордовой структурой $c$ и перестановочен с инволюциями $f_{1}$ и $f_{2}$ :

$$
\beta c(v)+c(v) \beta=0, \quad f_{i} \beta=\beta f_{i}, \quad i=1,2 .
$$

Прямое вычисление показьвает, что при замене ортонормированного базиса элемент $\beta$ изменяется на знак определителя матрицы перехода от одного базиса к другому. Следовательно, глобально этот элемент определяет изоморфизм векторных расслоений

$$
\beta: E \rightarrow E \otimes \Lambda^{2 k}(V)
$$

(которьй для краткости обозначается тем же символом). При этом коммутационные соотношения (30) переходят в

$$
\beta^{-1}\left(c \otimes 1_{\Lambda^{2 k}(V)}\right) \beta=-c, \quad \beta^{-1}\left(f_{i} \otimes 1_{\Lambda^{2 k}(V)}\right) \beta=f_{i}, \quad i=1,2 .
$$

Таким образом, изоморфность четверок

$$
\alpha^{*}\left[E, c, f_{1}, f_{2}\right] \quad \text { и } \quad\left[E, c, f_{1}, f_{2}\right] \otimes \Lambda^{2 k}(V)
$$

установлена. Теорема доказана. 
СлЕДСТВИЕ 3. Справедлива аналогичная формула для К-теории с коэффичиентами в $\mathbb{Z}_{n}$ :

$$
\alpha^{*}=(-1)^{n} \Lambda^{n}(V): K_{c}^{*}\left(V, \mathbb{Z}_{n}\right) \rightarrow K_{c}^{*}\left(V, \mathbb{Z}_{n}\right) .
$$

Действительно, $K$-теория с коэффициентами определяется при помощи пространства Мура $M_{n}$ по формуле

$$
K_{c}^{*}\left(V, \mathbb{Z}_{n}\right)=K_{c}^{*}\left(V \times M_{n}, V \times p t\right)
$$

Применяя доказанную теорему к пространству $X \times M_{n}$ и поднятию расслоения $V$ на него, получаем требуемую формулу в $K$-теории с коэффициентами.

ЗАмЕчАниЕ 5. В случае, когда пространство $X$ является гладким многообразием с кокасательньм расслоением $V=T^{*} X$, формула (29) указывает класс эллиптических символов, к которым произвольные символы могут быть приведены стабильными гомотопиями.

\section{5. Основная теорема}

ТЕОрема 9. Удвоенная дробная часть $\{2 d(\widehat{L})\}$ значения функиионала $d$ на подпространстве $\widehat{L}$ равна коэффициенту зацепления символа подпространства $c$ ориентирующим пучком $\Lambda^{n}(M), n=\operatorname{dim} M$, многообразия

$$
\{2 d(\widehat{L})\}=[L] \cap\left(1-\left[\Lambda^{n}(M)\right]\right) \in \mathbb{Z}\left[\frac{1}{2}\right] / \mathbb{Z} .
$$

ДоКАЗАТЕЛЬСТво. Напомним формулу (11) для дробной части функционала $d$ :

$$
\{2 d(\widehat{L})\}=\frac{1}{2^{N}} \bmod 2^{N} \text {-ind }\left[\left(1 \pm \alpha^{*}\right) \widehat{\sigma}\right], \quad[\sigma] \in K_{c}^{0}\left(T^{*} M, \mathbb{Z}_{2^{N}}\right)
$$

где $\sigma: 2^{N} L \rightarrow \pi^{*} F-$ изоморфизм векторных расслоений. Согласно теореме 8 из предыдущего раздела имеем

$$
\left(1 \pm \alpha^{*}\right)[\sigma]=\left[1-\Lambda^{n}(M)\right][\sigma]
$$

Поэтому

$$
\{2 d(\widehat{L})\}=\frac{1}{2^{N}} \bmod 2^{N} \text {-ind }\left[\left(1-\Lambda^{n}(M)\right) \widehat{\sigma}\right]
$$

что совпадает с определением индекса зацепления в разделе 3 , см. формулу (27).

Заметим также, что элемент $[L] \in K_{c}^{1}\left(T^{*} M\right)$ является элементом кручения в силу теоремы 4 , конечность порядка разности $\left[1-\Lambda^{n}(M)\right] \in K(M)$ доказывается в предложении 4. Теорема доказана.

СлЕДСТВИЕ 4. На ориентируемом многообразии функиионал размерности является полуцелочисленным, т.е.

$$
\{2 d(\widehat{L})\}=0
$$


ПРЕДЛОЖЕНИЕ 4. На неориентируемом многообразии $M$ размерности $2 k$ или $2 k+1$ имеет место следующая оченка для порядка дробной части инварианта $d$ :

$$
\left\{2^{k+1} d(\widehat{L})\right\}=0 .
$$

Аналогичные утверждения имеют место для $\eta$-инварианта.

ДОКАЗАТЕЛЬСТВО ПРЕДЛОЖЕНИЯ 4. Ориентирующий Пучок $\Lambda^{n}\left(M^{n}\right)$ является ОДномерным расслоением со структурной групой $\mathbb{Z}_{2}$. Следовательно, это векторное расслоение "снимается" с классифицирующего пространства $B \mathbb{Z}_{2}=\mathbb{R} \mathbb{P} \infty$, т.е. имеет место изоморфизм расслоений

$$
\Lambda^{n}\left(M^{n}\right) \simeq f^{*} \gamma \quad \text { для отображения } f: M^{n} \rightarrow \mathbb{R}^{N},
$$

здесь $\gamma$ - линейное расслоение прямых над проективным пространством $\mathbb{R P}^{N}$. По теореме о клеточной апроксимации можно выбрать $N=n$. Приведенные $K$-группы проективных пространств имеют вид

$$
\widetilde{K}\left(\mathbb{R}^{2 k}\right) \simeq \widetilde{K}\left(\mathbb{R}^{2 k+1}\right) \simeq \mathbb{Z}_{2^{k}} .
$$

Следовательно, выполнено равенство

$$
2^{k}\left(1-\left[\Lambda^{n}\left(M^{n}\right)\right]\right)=0 .
$$

Поэтому получаем требуемое

$$
\left\{2^{k+1} d(\widehat{L})\right\}=[L] \cap\left[2^{k}\left(1-\Lambda^{n}\left(M^{n}\right)\right)\right]=[L] \cap 0=0 .
$$

\section{6. Примеры}

1. Рассмотрим четномерное вещественное проективное пространство $\mathbb{R} \mathbb{P}^{2 n}$. Приведенная $K$-групша этого многообразия является циклической:

$$
\widetilde{K}\left(\mathbb{R P}^{2 n}\right) \simeq \mathbb{Z}_{2^{n}},
$$

а в качестве образуюшей можно выбрать ориентирующий пучок

$$
1-\left[\Lambda^{2 n}\left(\mathbb{R}^{2 n}\right)\right] \in \widetilde{K}\left(\mathbb{R P}^{2 n}\right) .
$$

$\mathrm{C}$ другой стороны, многообразие $\mathbb{R}^{2 n}$ имеет $\operatorname{pin}^{c}$ структуру, а главный символ самосопряженного $\operatorname{pin}^{c}$ оператора Дирака $D$ на нем (этот оператор построен в [3]) является образующей группы

$$
[\sigma(D)] \in K_{c}^{1}\left(T^{*} \mathbb{R}^{2 n}\right)=\operatorname{Tor} K_{c}^{1}\left(T^{*} \mathbb{R}^{2 n}\right) \simeq \mathbb{Z}_{2^{N}} .
$$

Из невырожденности формы зацепления следует, что для указанных образуюших имеет место равенство

$$
2^{n-1}[\sigma(D)] \cap\left[1-\left[\Lambda^{2 n}\left(\mathbb{R P}^{2 n}\right)\right]\right]=\frac{1}{2} .
$$

Поэтому $\eta$-инвариант pin $^{c}$ оператора Дирака $D$ имеет большую дробную часть (см. [3]):

$$
\left\{2^{n} \eta(D)\right\}=2^{n-1}[\sigma(D)] \cap\left[1-\left[\Lambda^{2 n}\left(\mathbb{R}^{2 n}\right)\right]\right]=\frac{1}{2} .
$$

Этот пример устанавливает точность оценки (31) знаменателя дробной части $\eta$-инварианта на четномерных многообразиях. 
2. Построим теперь оператор на нечетномерном многообразии с ненулевой дробной частью $\eta$-инварианта. Для этого воспользуемся конструкцией скрещенного произведения эллиптических операторов (ср. [18]).

Пусть $D_{1}$ - самосопряженньй эллиптический оператор на четномерном многообразии $M_{1}$ с нечетным символом:

$$
\sigma\left(D_{1}\right)(x,-\xi)=-\sigma\left(D_{1}\right)(x, \xi)
$$

a $D_{2}$ - эллиптический оператор на нечетномерном многообразии $M_{2}$ с символом, удовлетворяющим равенству

$$
\sigma\left(D_{2}\right)(x,-\xi)=\sigma\left(D_{2}\right)^{*}(x, \xi)
$$

(предполагается, что оператор $D_{2}$ действует в одном и том же расслоении). Через $M$ обозначим декартово произведение многообразий $M_{1} \times M_{2}$. Рассмотрим произведение

$$
\left[\sigma\left(D_{1}\right)\right] \times\left[\sigma\left(D_{2}\right)\right] \in \operatorname{Tor} K_{c}^{1}\left(T^{*} M\right)
$$

символов этих эллиптических операторов. Здесь

$$
\left[\sigma\left(D_{1}\right)\right] \in K_{c}^{1}\left(T^{*} M_{1}\right), \quad\left[\sigma\left(D_{2}\right)\right] \in K_{c}^{0}\left(T^{*} M_{2}\right) .
$$

Для (самосопряженного) символа $\sigma$ этого произведения имеет место формула

$$
\sigma(D)=\left(\begin{array}{cc}
\sigma\left(D_{1}\right) \otimes 1 & 1 \otimes \sigma\left(D_{2}\right)^{*} \\
1 \otimes \sigma\left(D_{2}\right) & -\sigma\left(D_{1}\right) \otimes 1
\end{array}\right) .
$$

Вычислим индекс зацепления элемента $\left[\sigma\left(D_{1}\right)\right] \times\left[\sigma\left(D_{2}\right)\right]$ с ориентирующим пучком произведения $M_{1} \times M_{2}$.

ПРЕДЛОЖЕНИЕ 5. Пусть многообразие $M_{2}$ является ориентируемым. Тогда имеет место формула

$\left(\left[\sigma\left(D_{1}\right)\right] \times\left[\sigma\left(D_{2}\right)\right]\right) \cap\left(1-\Lambda^{n}\left(M^{n}\right)\right)=\left[\sigma\left(D_{1}\right)\right] \cap\left(1-\Lambda^{k}\left(M_{1}\right)\right)$ ind $D_{2}, \quad \operatorname{dim} M_{1}=k$.

ДокАЗАТЕЛЬСтво. Рассмотрим коммутативную диаграмму

$$
\begin{array}{ll}
K_{c}^{0}\left(T^{*} M_{1}, \mathbb{Q} / \mathbb{Z}\right) \times K_{c}^{0}\left(T^{*} M_{2}\right) \longrightarrow K_{c}^{0}\left(T^{*} M, \mathbb{Q} / \mathbb{Z}\right) \\
\cap\left(1-\Lambda^{k}\left(M_{1}\right)\right) \times 1 \uparrow & \cap\left(1-\Lambda^{k}\left(M_{1}\right)\right) \uparrow \\
\operatorname{Tor} K_{c}^{1}\left(T^{*} M_{1}\right) \times K_{c}^{0}\left(T^{*} M_{2}\right) \longrightarrow \operatorname{Tor} K_{c}^{1}\left(T^{*} M\right)
\end{array}
$$

Здесь отображения, действующие по горизонтали, являются умножениями в $K$-теории. В силу ориентируемости второго многообразия имеем

$$
1-\left[\Lambda^{k}\left(M_{1}\right)\right]=1-\left[\Lambda^{n}(M)\right] .
$$

Поэтому формула (34) вытекает из этой диаграммы применением прямого образа

$$
p_{!}: K_{c}^{0}\left(T^{*} M, \mathbb{Q} / \mathbb{Z}\right) \rightarrow \mathbb{Q} / \mathbb{Z}
$$

Предложение доказано. 
ЗАмЕчАниЕ 6. Формула (34) является аналогом известного свойства $\eta$-инварианта (см. [2]): $\eta$-инвариант скрещенного произведения операторов равен произведению $\eta$-инварианта первого сомножителя на индекс второго.

K сожалению, расслоение $L_{+}(\sigma(D))$ не является четным. В самом деле, для символа $\sigma$ справедливо равенство

$$
\alpha^{*} \sigma=\left(\begin{array}{ll}
0 & 1 \\
1 & 0
\end{array}\right) \sigma\left(\begin{array}{ll}
0 & 1 \\
1 & 0
\end{array}\right) .
$$

Следовательно, для спектрального подпространства имеем

$$
\alpha^{*} L_{+}(\sigma)=\left(\begin{array}{ll}
0 & 1 \\
1 & 0
\end{array}\right) L_{+}(\sigma)
$$

Однако это расслоение изоморфно четному расслоению.

ПРЕДЛОЖЕНИЕ 6. Существует такое векторное расслоение $L \in \operatorname{Vect}\left(P^{*} M\right)$ над расслоением проективных пространств, что его поднятие $p^{*} L$ на косферы $S^{*} M$ при проекчии $p: S^{*} M \rightarrow P^{*} M$ изоморфно расслоению $L_{+}(\sigma)$.

$$
S(V \oplus 1)
$$

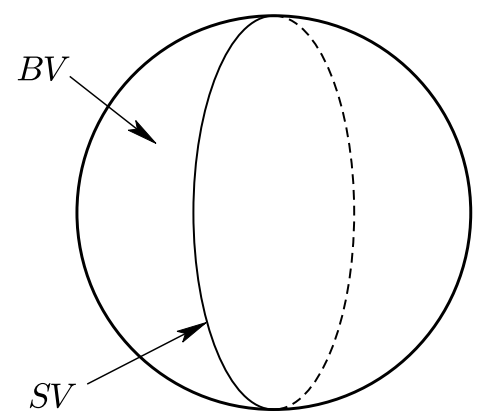

$$
P^{*} M=B V / v \sim-v
$$
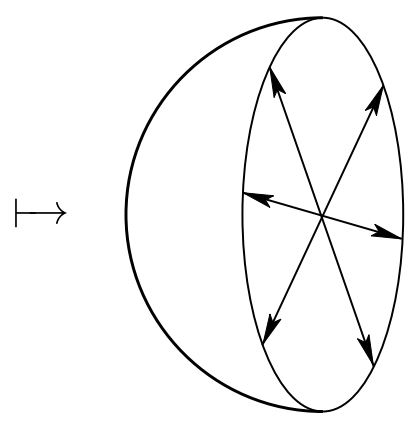

Рис. 1. Расслоение проективных пространств $P^{*} M$

ДокАЗАтЕльство. Фиксируем на нечетномерном многообразии $M$ ненулевое векторное поле. Рассмотрим отвечающее ему разложение кокасательного расслоения $T^{*} M=V \oplus 1$ (относительно некоторой римановой метрики). Тогда для косферического расслоения имеем

$$
S^{*} M=S(V \oplus 1)
$$

а его проективизация $P^{*} M$ диффеоморфна расслоению единичных шаров

$$
B V \subset S(V \oplus 1)
$$

расслоения $V$ с отождествленньми антиподальными точками на границе:

$$
P^{*} M=B V /\{v \sim-v|| v \mid=1\}
$$


(см. рис. 1). Тогда расслоение $L$ над проективизацией $P^{*} M$ получается аналогичньм образом из расслоения $L_{+}(\sigma)$ над шарами $B V$ при помощи отождествления слоев над антиподальньми точками $\pm v$ при помощи инволюции

$$
\left(\begin{array}{ll}
0 & 1 \\
1 & 0
\end{array}\right)
$$

Изоморфность поднятия расслоения $L$ на сферы $S^{*} M$ и исходного расслоения $L_{+}(\sigma)$ проверяется непосредственно. Предложение доказано.

Из этих двух утверждений получаем

СлЕДСТВИЕ 5. Для четного подпространства $\widehat{L}$ с построенным символом $L$ имеет место равенство

$$
\{2 d(\widehat{L})\}=\left\{2 d\left(\widehat{L}_{+}\left(D_{1}\right)\right)\right\} \text { ind } D_{2}
$$

3. Применим полученную формулу в следующем частном случае. Положим $M_{1}=$ $\mathbb{R P}^{2 k}, D_{1}-\operatorname{pin}^{c}$ оператор Дирака из первого примера. В качестве второго многообразия рассмотрим окружность $M_{2}=S^{1}$, на которой фиксируем псевдодифференциальный оператор $D_{2}$ с главным символом

$$
\sigma\left(D_{2}\right)(\varphi, \xi)= \begin{cases}e^{-i \varphi}, & \xi=1 \\ e^{i \varphi}, & \xi=-1\end{cases}
$$

Имеем ind $D_{2}=2$ и функционал размерности четного подпространства $\widehat{L}$, получаемого в силу предложения 6, имеет ненулевую дробную часть; более точно,

$$
\left\{2^{k-1} d(\widehat{L})\right\}=\frac{1}{2}
$$

Отметим также одно следствие. Оно дает ответ на вопрос, поставленньй в [1].

СлЕдСТВИЕ 6. Существуют дифференциальные операторы четного порядка на нечетномерных многообразиях с произвольной двоично-рачиональной дробной частью п-инварианта.

4. Рассмотрение дифференциальных операторов порядка выше первого может показаться экзотикой для теории индекса, однако известны геометрические операторы второго порядка с интересными спектральными свойствами (см. [1]). Следует также упомянуть, что оператор Хирцебруха на ориентированном многообразии эквивалентен оператору второго порядка. В частности, сигнатура многообразия равна индексу оператора второго порядка. Это наблюдение позволило Конну, Телеману и Сулливану [24] выразить сигнатуру липшицевых и квазиконформных многообразий как индекс ограниченного фредгольмового оператора. 


\section{СПИСОК ЦИТИРОВАННОЙ ЛИТЕРАТУРЫ}

[1] Gilkey P. B. The eta invariant of even-order operators // Lecture Notes in Math. 1989. V. 1410. P. 202-211.

[2] Atiyah M., Patodi V., Singer I. Spectral asymmetry and Riemannian geometry, I // Math. Proc. Cambridge Phil. Soc. 1975. V. 77. P. 43-69; II // 1976. V. 78. P. 405-432; III // 1976. V. 79. P. 315-330.

[3] Gilkey P. B. The eta invariant for even dimensional Pin ${ }^{c}$ manifolds // Advances in Math. 1985. V. 58. P. 243-284.

[4] Bahri A., Gilkey P. The eta invariant, $\operatorname{Pin}^{c}$ bordism, and equivariant $\operatorname{Spin}^{c}$ bordism for cyclic 2-groups // Pacific J. Math. 1987. V. 128. №1. P. 1-24.

[5] Pontrjagin L. S. Über den algebraischen Inhalt topologischer Dualitatssatze // Math. Ann. 1931. V. 105. № 2. P. 165-205.

[6] Понтрягин Л.С. Топологические теоремы двойственности // УМН. 1947. Т. 2. № 2. C. $21-44$.

[7] Seifert H., Threllfall W. Lehrbuch der Topologie. Leipzig: Teubner, 1934.

[8] Фоменко А., Фукс Д. Курс гомотопической топологии. М.: Наука, 1989.

[9] Milnor J. Torsion et type simple d'homotopie // Essays Topol. Relat. Top. Mem. dédiés à Georges de Rham, 1970. P. 12-17.

[10] Brumfiel G., Morgan J. Quadratic functions, the index modulo 8, and a $\mathbb{Z} / 4$-Hirzebruch formula // Topology. 1973. V. 12. P. 105-122.

[11] Kervaire M., Milnor J. Groups of homotopy spheres, I // Ann. Math. 1963. V. 77. №3. P. 504-537.

[12] Moore G., Witten E. Self-duality, Ramond-Ramond fields and $K$-theory // E-print hep-th/9912279, 1999.

[13] Савин А. Ю., Стернин Б. Ю. Эллиптические операторы в четных подпространствах // Матем. сб. 1999. Т. 190. № 8. С. 125-160.

[14] Savin A. Yu., Sternin B. Yu. Elliptic Operators in Odd Subspaces. Preprint № 99/11 (Juni 1999). Potsdam: Univ. Potsdam, Institut für Mathematik, 1999; E-print math.DG/9907039.

[15] Savin A., Schulze B.-W., Sternin B. Elliptic Operators in Subspaces. Preprint № 00/04 (Februar 2000). Potsdam: Univ. Potsdam, Institut für Mathematik, 2000.

[16] Savin A., Schulze B.-W., Sternin B. Elliptic Operators in Subspaces and the Eta Invariant. Preprint № 99/14 (Juni 1999). Potsdam: Univ. Potsdam, Institut für Mathematik, 1999; E-print math.DG/9907047.

[17] Karoubi M. K-Theory. An Introduction. Grundlehren Math. Wiss. V. 226. Berline: Springer-Verlag, 1978.

[18] Atiyah M.F., Singer I. M. The index of elliptic operators, I // Ann. of Math. 1968. V. 87. P. 484-530.

[19] Бирман М. С., Соломяк М.З. О подпространствах, допускающих псевдодифференциальный проектор // Вестн. ЛГУ. 1982. № 1. С. 18-25.

[20] Bott R., Tu L. Differential Forms in Algebraic Topology. Graduate Texts in Math. V. 82. Berlin-Heidelberg-New York: Springer-Verlag, 1982.

[21] Baum P., Douglas R. G. Toeplitz operators and Poincaré duality // Toeplitz Centennial. Proceedings of Toeplitz Memory Conf. (Tel Aviv, 1981). Operator Theory Adv. Appl. 1982. V. 4. P. 137-166.

[22] Karoubi M. Algèbres de Clifford et $K$-theorie // Ann. Sci. Ecole Norm. Sup. 1968. V. 4. № 1. P. 161-270.

[23] Мищенко А. С. Векторные расслоения и их применения. М.: Наука, 1984.

[24] Connes A., Sullivan D., Teleman N. Quasiconformal mappings, operators on Hilbert space, and local formulae for characteristic classes // Topology. 1994. V. 33. № 4. P. 663-681.

Московский государственный университет им. М.В.Ломоносова

Поступило

E-mail: antonsavin@mtu-net.ru, sternine@mtu-net.ru 25.04 .2001 